\title{
Effect of CCK-8 on the expression of Bax, Bcl-2 and Caspase- 3 of the hippocampus CA1 neurons in morphine in relapsed rats
}

\author{
Guanghua Ye ${ }^{1,2,3}$, Chunling $\mathrm{Ma}^{4}$, Yuan Gao ${ }^{1,3}$, Linsheng Yu ${ }^{1,3}$, Yanyan Fan ${ }^{1,3}$, Haiyan $\mathrm{Hu}^{1,3}$, Xiping Chen ${ }^{2}$, Luyang Tao ${ }^{2 *}$ and Feng $\mathrm{Li}^{1,3,5 *}$ \\ ${ }^{1}$ Department of Forensic Medicine, Wenzhou Medical University, Wenzhou, Zhejiang, China \\ ${ }^{2}$ Institute of Forensic Sciences, Soochow University, Suzhou, Jiangsu, China \\ ${ }^{3}$ Institute of Forensic Science, Wenzhou Medical University, Wenzhou, Zhejiang, China \\ ${ }^{4}$ Hebei Key Laboratory of Forensic Medicine, Department of Forensic Medicine, Hebei Medical University, PR China \\ ${ }^{5}$ Forensic Medical Management Services, 850 R. S. Gass Blvd, Nashville, TN, 37216, China
}

\begin{abstract}
Objective: To explore the neuroprotective mechanisms of CCK- 8 for morphine relapse by researching the effect of CCK- 8 on the expression of Bax, Bcl-2 and Caspase- 3 of hippocampus CA1 neurons in morphine-relapsed rats.

Methods: A total of 40 healthy male Wistar rats (weight 200g $\pm 10 \mathrm{~g}$ ) were divided into the following groups: normal saline (NS) group ( $\mathrm{n}=8$ ); morphine (MOR) group $(n=8)$; and CCK-8 treated group $(n=24)$. The groups were selected by the random peer-to-peer principle. The CCK- 8 treated group was subdivided into $0.01 \mu \mathrm{g}$ CCK-8 group $(\mathrm{n}=8), 0.1 \mu \mathrm{g}$ CCK-8 group $(\mathrm{n}=8)$ and $1.0 \mu \mathrm{g}$ CCK-8 group $(\mathrm{n}=8)$. First we established the models of morphine-relapsed rats under CCK-8 intervention using the Conditioned Place Preference (CPP) methods. Three different doses of CCK-8 (0.01, 0.1 and $1.0 \mu$ g, i.c.v) were injected 15 minutes before morphine administration during the conditioning phase. All rats were decapitated and their hippocampi CA1 were made into slices with immunohistochemical stains. The expressions of $\mathrm{Bax}, \mathrm{Bcl}-2$, Caspase- 3 of neurons were then measured by their ODs, and each ratio of Bax and Bcl-2 (Bax/Bcl-2) were calculated using a microscope connected to a computer equipped with Image-ProPlus software.
\end{abstract}

Results: In the NS group, the expression of Bcl-2 was very weak and the expression of Bax and Caspase-3 was barely observed. In the MOR group, the expression of Bax and Caspase- 3 was strong and that of $\mathrm{Bcl}-2$ was weak, and there were significant differences $(\mathrm{P}<0.05)$ compared to the NS group. As to Bax, Caspase- 3 the ratio of $\mathrm{Bax} / \mathrm{Bcl}-2$, showed a gradually decreasing trend in the sequence of group $0.01 \mu \mathrm{g}$, group $0.1 \mu \mathrm{g}$, and group $1.0 \mu \mathrm{g}$, but when it came to that of $\mathrm{Bcl}-2$, it was opposite. There were significant differences $(\mathrm{P}<0.05)$, compared to the MOR group, except in group $0.01 \mu \mathrm{g}$.

Conclusion: CCK-8 could up regulate the expression of Bcl-2, down regulate Bax and Caspase-3 as well as reduce the Bax/ Bcl-2 ratio of neurons of hippocampus CA1 in the model of morphine-relapsed rats. This may be one of the main neuroprotective mechanisms of CCK-8 for morphine relapse.

\section{Introduction}

Morphine addiction is a worldwide problem resulting in increasing damage to an individual's health and social stability. Presently morphine addiction can be basically cured by "stupor" through the use of "methadone recursive subtraction." For those patients who use methadone for 7 to 10 days, more than $95 \%$ of cured patients will relapse within half a year [1]. The high rate of relapse becomes a major problem for dealing with drug addiction [2]. Clinical data showed that once individuals become addicted, a majority of them are trapped in the vicious ciycle of dependence, detoxification, relapse, re-dependence, re-detoxification and relapse. Thus the key to break this cycle is protecting addicts from relapsing, which is the essential focus of this study.

Liu LW, et al., [3] reported that morphine can induce neuronal apoptosis in the brain by increasing the expressions of pro-apoptotic Fas and Caspase- 3 and decreasing the anti-apoptotic Bcl-2 expression. CCK-8, an anti-opioid peptide, is the most potent neuropeptide which has been shown to play a key role in the central nervous system. Our previous research displayed that pretreatment with CCK-8 significantly inhibited the acquisition of morphine induced
Conditioned Place Preference (CPP) in rats [4], which demonstrated that CCK- 8 could protect the brain from morphine relapse. Therefore, the neuroprotective mechanisms of whether or not CCK- 8 protects the brain by anti-apoptosis need to be further ascertained. We are investigating some anti-apoptotic neuroprotective mechanisms of CCK-8 for morphine relapse.

\section{Animals and drugs}

Forty (40) healthy male Wistar rats were obtained from the Center of Laboratory Animal Science at the Hebei Medical University. Animal

Correspondence to: Luyang Tao, Institute of Forensic Sciences, Soochow University, Suzhou, Jiangsu, China; E-mail: taoluyang@suda.edu.cn and Feng Li, Forensic Medical Management Services, 850 R. S. Gass Blvd, Nashville, TN, 37216, China, E-mail: fli@forensicmed.com

Key words: Cholecystokinin-8, Morphine, Bax, Bcl-2, Caspase-3, Morphine relapse Received: February 03, 2018; Accepted: February 26, 2018; Published: February 28,2018 
care and the experimental procedures were conducted according to the National Institutes of Health Guide for the Care and Use of Laboratory Animals. The rats weighed $200 \mathrm{~g} \pm 10 \mathrm{~g}$. All protocols in this study were approved by the Local Committee of Animal Use and Protection of the Hebei Medical University. Morphine hydrochloride was obtained from Shenyang First Pharmaceutical Factory (Liaoning, China). CCK-8 was purchased from Sigma, Ltd. (MA, USA). CCK-8 was suspended to a concentration of $1 \mathrm{mg} / \mathrm{ml}$ in $1 \%$ ammonia saline solution. The working solution of CCK-8 was then diluted in saline to the concentrations of $0.5,0.05$ and $0.005 \mathrm{mg} / \mathrm{ml}$ immediately before use.

\section{Procedures}

All rats were divided into normal saline group (NS, $n=8)$, morphine group (MOR, $n=8$ ), and a CCK- 8 treated group $(n=24)$ by the random peer-to-peer principle. The CCK- 8 treated group was subdivided into $0.01 \mu \mathrm{g}$ CCK-8 group $(0.01 \mu \mathrm{g}, \mathrm{n}=8), 0.1 \mu \mathrm{g}$ CCK-8 group $(0.1 \mu \mathrm{g}, \mathrm{n}=8)$ and $1.0 \mu \mathrm{g}$ CCK- 8 group $(1.0 \mu \mathrm{g}, \mathrm{n}=8)$. Based on our previous research [5] we established the models of morphine-relapse rats under CCK-8 intervention using the CPP methods. Three different doses of CCK$8(0.01,0.1$ and $1.0 \mu$ g, i.c.v $)$ were intracerebroventricularly injected 15 minutes before intraperitoneal administration of morphine during the conditioning phase. All rats were decapitated and their areas of hippocampus CA1 were made into slices and immunohistochemically stained. The expressions of Bax, Bcl-2, Caspase-3 of neurons in hippocampus CA1 area were measured by computing their average optical densities (ODs) of positive cells with an Image-Pro Plus 6.0 image analytical software under the microscope. Also, each ratio of Bax and $\mathrm{Bcl}-2(\mathrm{Bax} / \mathrm{Bcl}-2)$ was calculated.

\section{Statistical analysis}

The data were analyzed with the statistical software SPSS 18.0 using a one-way ANOVA. The measurement data of normal distribution were expressed with mean \pm standard deviation $(\mathrm{x} \pm \mathrm{s})$, analysis of variance was applied when comparing groups and a LSD-t test was applied when making a comparison between two groups. The difference was statistically significant if the $\mathrm{P}$ values were found to be $<0.05$.

\section{Results}

In the NS group the expression of Bcl-2 was very weak and that of Bax and Caspase- 3 was barely observed. In the MOR group, Bax and Caspase- 3 were strong and $\mathrm{Bcl}-2$ was weak, and when compared to the NS group, the differences were significant $(\mathrm{P}<0.05)$. As to the $\mathrm{Bax}$, Caspase- 3 and the ratio of $\mathrm{Bax} / \mathrm{Bcl}-2$, there was a gradually decreasing trend in the sequence of CCK- 8 treated group $0.01 \mu \mathrm{g}$, group $0.1 \mu \mathrm{g}$, group $1.0 \mu \mathrm{g}$, but when it came to Bcl-2, it was opposite. There were significant differences $(\mathrm{P}<0.05)$ when compared to the MOR group except for the group $0.01 \mu \mathrm{g}$. (Table 1, Figures 1-5).

Protein expression of Bcl-2 was very weak and that of Bax and Caspase- 3 was barely observed in the NS Group. Bax and Caspase- 3 were strong and $\mathrm{Bcl}-2$ was weak in the MOR group and when compared

Table 1. OD of Bax, Bcl-2, Caspase-3 expression in rats' hippocampal CA1 neurons $(\mathrm{n}=8, \bar{x} \pm \mathrm{s})$

\begin{tabular}{|c|c|c|c|c|}
\hline Group & Bax & Bcl-2 & Bax/ Bcl-2 & Caspase-3 \\
\hline NS & 0.001 & $0.018 \pm 0.008$ & 0.001 & 0.001 \\
\hline MOR & $0.352 \pm 0.005^{\#}$ & $0.095 \pm 0.004^{\#}$ & $3.756 \pm 0.457^{\#}$ & $0.325 \pm 0.03^{\#}$ \\
\hline $0.01 \mu \mathrm{g}$ & $0.316 \pm 0.002$ & $0.110 \pm 0.002$ & $2.883 \pm 0.103$ & $0.305 \pm 0.001$ \\
\hline $0.1 \mu \mathrm{g}$ & $0.113 \pm 0.00^{5} \$$ & $0.271 \pm 0.007^{\$}$ & $0.417 \pm 0.033 \$$ & $0.104 \pm 0.001^{\$}$ \\
\hline $1.0 \mu \mathrm{g}$ & $0.103 \pm 0.004^{\$}$ & $0.313 \pm 0.008^{\S}$ & $0.331 \pm 0.050^{\$}$ & $0.097 \pm 0.004^{\$}$ \\
\hline \# Compared to NS P $<0.05 ; \$$ Compared to MOR P $<0.05$ \\
\hline
\end{tabular}

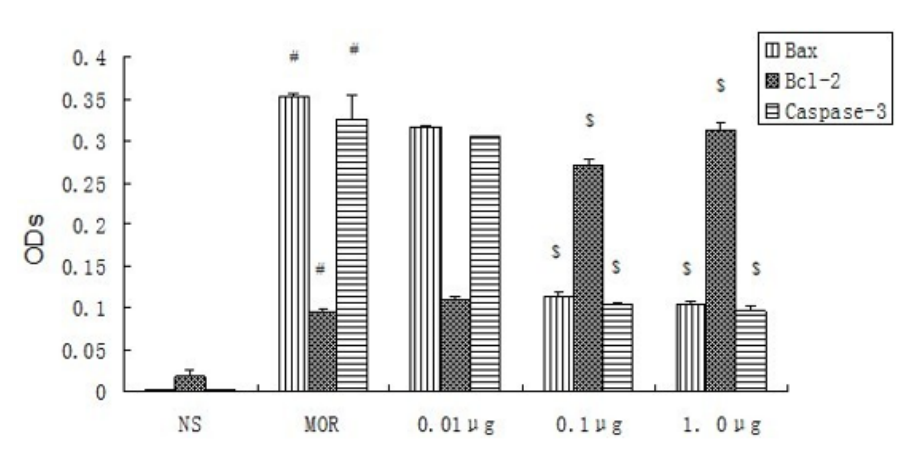

Figure 1. OD of Bax, Bcl-2, Caspase-3 expression in rats' hippocampal CA1 neurons $(\mathrm{n}=8, \pm \mathrm{s})$

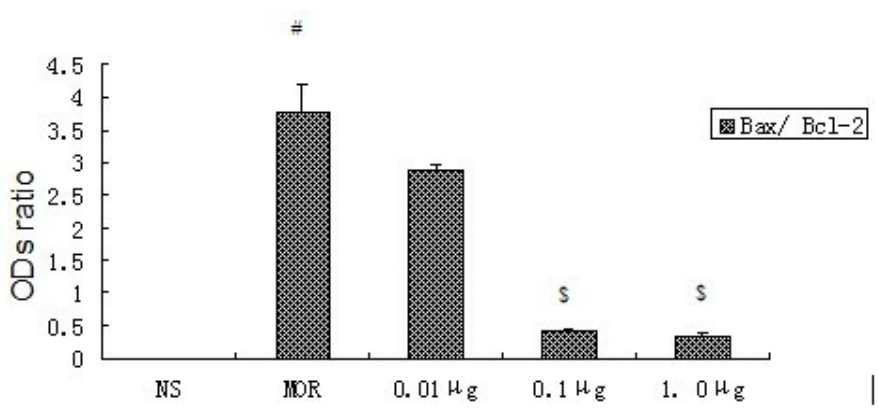

Figure 2. Ratio of ODs of Bax and Bcl-2 expression in rats' hippocampal CA1 neurons $(\mathrm{n}=8, \pm \mathrm{s})$.

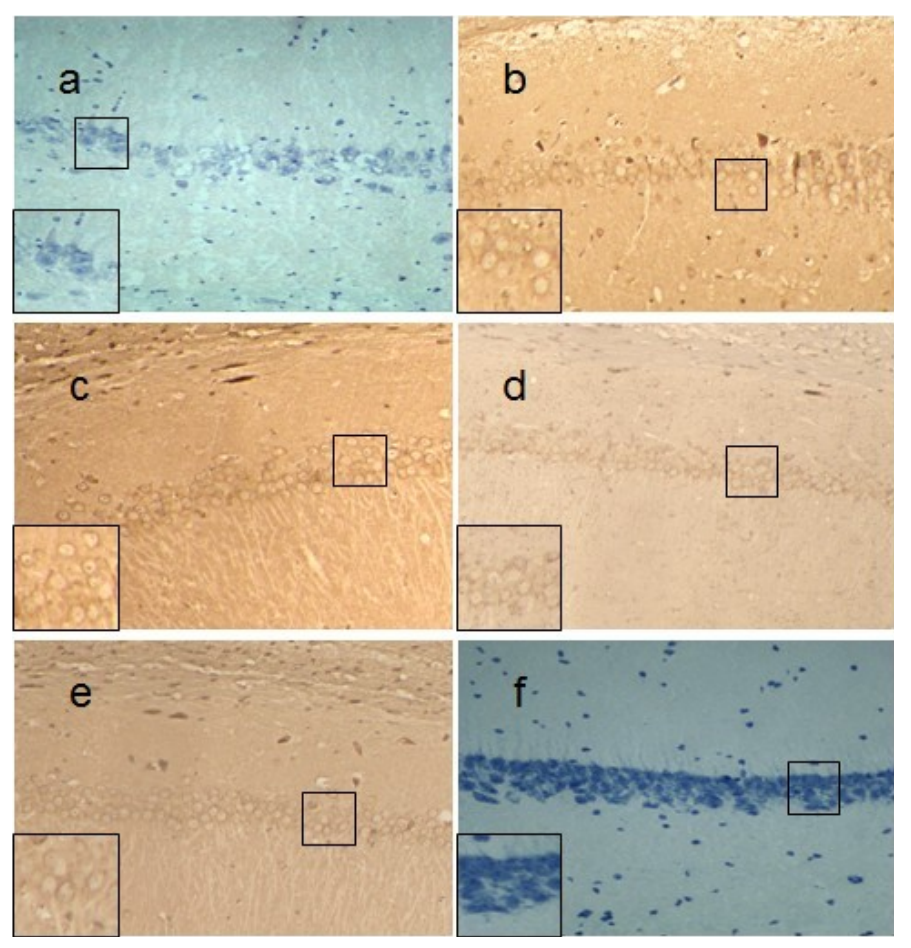

Figure 3. Bax expression of different groups in rats' hippocampal CA1 neurons $(\mathrm{SP} \times 200)$. a: expression of Bax was barely observed in the NS Group; b: expression of Bax was strongest in the MOR group; c: expression of Bax was strong in group $0.01 \mu \mathrm{g}$; d: expression of Bax was a little weak in group 0.1 $\mu \mathrm{g}$; e: expression of Bax was weak in group 1. $0 \mu \mathrm{g}$; f: Negative control.

to the NS Group the differences were significant $(\mathrm{P}<0.05)$. As to Bax, Caspase- 3 , there is a gradually decreased trend in the sequence of CCK-8 treated group $0.01 \mu \mathrm{g}$, group $0.1 \mu \mathrm{g}$ and group $1.0 \mu \mathrm{g}$, but when it comes to that of $\mathrm{Bcl}-2$, it was opposite. There were significant 


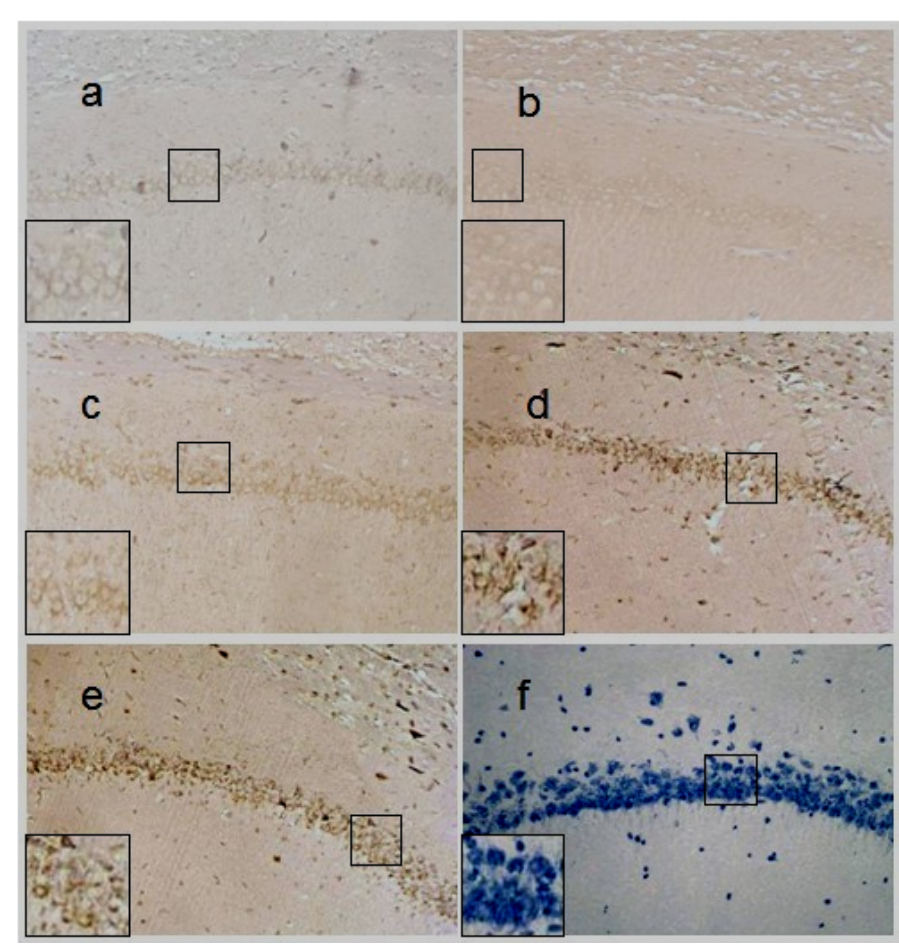

Figure 4. Bcl-2 expression of different groups in rats' hippocampal CA1 neurons $(\mathrm{SP} \times 200)$. a: expression of Bcl-2 was weak in Group NS; b: expression of Bcl-2 was a little strong in group MOR; c: expression of Bcl-2 was strong in CCK-8 treated group $0.01 \mu \mathrm{g}$; $\mathrm{d}$ : expression of Bcl-2 was strong in CCK-treated group 0.1 $\mu \mathrm{g}$; e: expression of Bcl-2 was strongest in CCK-treated group 1. $0 \mu \mathrm{g}$; f: Negative control.

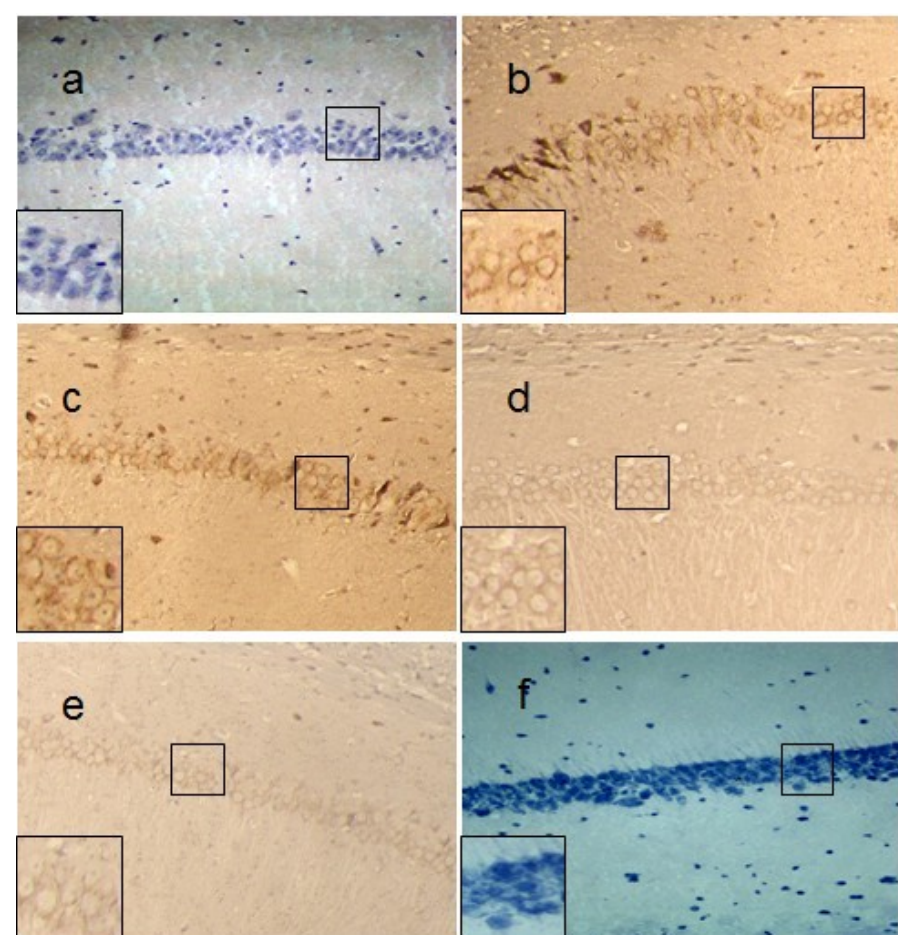

Figure 5. Caspase-3 expression of different groups in rats' hippocampal CA1 neurons $(\mathrm{SP} \times 200)$. a: expression of Caspase- 3 was barely observed in the NS Group; b: expression of Caspase-3 was strongest in the MOR group; c: expression of Caspase-3 was strong in CCK-treated group $0.01 \mu \mathrm{g}$; d: expression of Caspase- 3 was a little weak in CCK-8 treated group $0.1 \mu \mathrm{g}$; e: expression of Caspase-3 was weak in CCK-treated group 1. $0 \mu \mathrm{g}$; f: Negative control.

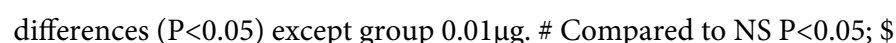
Compared to MOR $\mathrm{P}<0.05$.

There is a gradual decreased trend about the ratio of Bax/ Bcl-2 in the sequence of group MOR, CCK-treated group $0.01 \mu \mathrm{g}$, group $0.1 \mu \mathrm{g}$ and group $1.0 \mu \mathrm{g}$. There were significant differences $(\mathrm{P}<0.05)$, compared to Group MOR, except group $0.01 \mu \mathrm{g}$. \# Compared to NS $\mathrm{P}<0.05$; \$ Compared to MOR $\mathrm{P}<0.05$.

\section{Conclusion}

CCK-8 could up-regulate the expression of Bcl-2, down-regulate that of Bax and Caspase- 3 and reduce the $\mathrm{Bax} / \mathrm{Bcl}-2$ ratio of neurons of hippocampus CA1 in the model of morphine-relapsed rats. This may be indicative of one of the main neuroprotective mechanisms of CCK- 8 for morphine relapse.

\section{Discussion}

There were many researchers [6,7] who have reported that morphine could induce apoptosis of neurons. There were many other research studies [8,9] which have reported that downregulation or antagonist of the CCK receptor promotes apoptosis, which indicates that CCK may inhibit apoptosis. Our previous research $[4,5,10]$ indicated CCK-8 could effectively improve all kinds of morphineaddicted behavioral functions. Therefore we hypothesized that CCK- 8 may protect neurons by inhibiting apoptosis in morphine-relapsed rats and our research has endeavored to seek the evidence of the same.

In this study, a perfect morphine-relapsed model with successful CCK-8 injection was established based on our previous research $[4,5,10]$, then the slices with immunohistochemically stained hippocampus CA1 were made. The expressions of Bax, Bcl-2, Caspase-3 and each ratio of $\mathrm{Bax}$ and $\mathrm{Bcl}-2$ were scientifically measured and statistically analyzed. Bax and Bcl-2 are the major apoptosis regulating genes which control apoptotic pathways at the upstream [11]. They have completely opposite functions. Bax promotes apoptosis but Bcl-2 inhibits apoptosis, and the $\mathrm{Bax} / \mathrm{Bcl}-2$ ratio eventually decides whether apoptosis or not [12]. Caspase-3 is the most important apoptosisregulating gene at the downstream which controls the last gateway of apoptotic pathway [13]. Thus the detection of expression of Bax, Bcl2 , caspase- 3 and calculation of $\mathrm{Bax} / \mathrm{Bcl}-2$ ratio could precisely reflect apoptosis [14]. Our experimental results showed that CCK-8 could upregulate the expression of $\mathrm{Bcl}-2$ and down-regulate Bax and Caspase- 3 and reduce $\mathrm{Bax} / \mathrm{Bcl}-2$ ratio, which illustrated that CCK- 8 could protect neurons by inhibiting apoptosis in morphine-relapsed rats.

As a postscript, this study just focused on whether or not CCK8 exerted influences on expressions of $\mathrm{Bax}, \mathrm{Bcl}-2$ and Caspase- 3 of hippocampus CA1 neurons in morphine-relapsed rats. But the mechanisms that just how CCK- 8 specifically exerts influences on them need further study.

\section{Conflicts of interest}

No conflict of interest to declare.

\section{Acknowledgement}

We gratefully acknowledge the generous assistance from the National Natural Science Foundation of China (No. 81172911, No. 81271379, No. 81301039, No. 81530062, No. 81273337, No.81172900); National High Technology Research and Development Program of China (863 Program, No.2015AA020503); and the Wenzhou Science and Technology Public Welfare Project (Y20150112). 


\section{References}

1. Stewart J (2003) Stress and relapse to drug seeking: studies in laboratory animals shed light on mechanisms and sources of long- term vulnerability Am J Addict 12: 1-17. [Crossref]

2. Smyth BP, Barry J, Keenan E, Ducray K (2010) Lapse and relapse following inpatient treatment of opiate dependence Ir Med J 103: 176-179. [Crossref]

3. Liu LW, Lu J, Wang XH, Fu SK, Li Q, et al. (2013) Neuronal apoptosis in morphine addiction and its molecular mechanism. Int J Clin Exp Med 6: 540-545. [Crossref]

4. Wen D, Cong B, Ma C, Yang S, Yu H, et al. (2012) The effects of exogenous CCK-8 on the acquisition and expression of morphine induced CPP. Neurosci Lett 510: 24-28. [Crossref]

5. Wen D, Zang G, Sun D, Yang S, Yu F, et al. (2013) Effects of cck-8 on the reinstatement of morphine induced $\mathrm{CPP}$ and expression of behavioral sensitization in rats. Neuroscience 238: 230-241. [Crossref]

6. Shafie A, Moradi F, Izadpanah E, Mokarizadeh A, Moloudi MR, et al. (2015) Neuroprotection of donepezil against morphine-induced apoptosis is mediated through Toll-like receptors. Eur J Pharmacol 764: 292-297. [Crossref]

7. Motaghinejad M, Karimian M, Motaghinejad O, Shabab B, Yazdani I, et al. (2015) Protective effects of various dosage of Curcumin against morphine induced apoptosis and oxidative stress in rat isolated hippocampus. Pharmacol Rep. 67: 230-235. [Crossref]
8. Fino KK, Matters GL, McGovern CO, Gilius EL, Smith JP (2012) Downregulation of the CCK-B receptor in pancreatic cancer cells blocks proliferation and promotes apoptosis. Am J Physiol Gastrointest Liver Physiol 302: G1244-1252. [Crossref]

9. Carrillo J, Agra N, Fernández N, Pestaña A, Alonso J (2009) Devazepide, a nonpeptide antagonist of CCK receptors, induces apoptosis and inhibits Ewing tumor growth. Anticancer Drugs 20: 527-533. [Crossref]

10. Yang S, Wen D, Dong M, Li D, Sun D, et al. (2013) Effects of cholecystokinin-8 on morphine-induced spatial reference memory impairment in mice. Behav Brain Res 256 : 346-53. [Crossref]

11. Hu XL, OlssonT, Johansson IM, Brännström T, Wester P (2004) Dynamic changes of the anti-and- pro-apoptotic proteins Bcl-w, Bcl-2, and Bax with Smac/ Diablomitochondrial release after photothrom-boticring stroke in rats. Eur J Neurosci 20: 1177-1188. [Crossref]

12. Almeida OF, Condé GL, Crochemore C, Demeneix BA, Fischer D, et al. (2000) Subtle shifts in the ratio between pro- and antiapoptotic molecules after activation of corticosteroid receptors decide neuronal fate. FASEB $J$ 14: 779-790. [Crossref]

13. Mazumder S, Plesca D, Almasan A (2008) Caspase-3 activation is a critical determinant of genotoxic stress-induced apoptosis. Methods Mol Biol 414: 13-21. [Crossref]

14. Ryu B, Ahn BN, Kang KH, Kim YS, Li YX, et al. (2015) Dioxinodehydroeckol protects human keratinocyte cells from UVB-induced apoptosis modulated by related genes Bax/Bcl-2 and caspase pathway. J Photochem Photobiol B 153: 352-357. [Crossref]

Copyright: (C2018 Ye G. This is an open-access article distributed under the terms of the Creative Commons Attribution License, which permits unrestricted use, distribution, and reproduction in any medium, provided the original author and source are credited. 